\title{
Supervisión de casos: Mujer embarazada traumatizada
}

\author{
Carol Forgash \\ Smithtown, NY \\ Andrew Leeds \\ Santa Rosa, CA \\ Claire A. I. Stramrood \\ Centro Médico Universitario, Groningen, Holanda \\ Amy Robbins \\ Atlanta, GA
}

\begin{abstract}
Supervisión de casos es un nuevo apartado regular del Journal of EMDR Practice and Research en el que un terapeuta solicita ayuda con un caso complicado y tres expertos le responden por escrito. En este artículo, Amy Robbins, una terapeuta certificada en terapia de desensibilización y reprocesamiento por movimientos oculares (EMDR) de Atlanta, Georgia, describe brevemente un caso complicado en el que una mujer embarazada busca tratamiento por un trauma sufrido durante un tornado. La terapeuta pregunta si es recomendable hacer tratamiento con EMDR y qué precauciones debería tener en cuenta. La primera especialista, Carol Forgash, ofrece información general sobre el embarazo y la psicoterapia y señala aspectos a tener en cuenta, precauciones y contraindicaciones a la hora de proceder con EMDR. Recomienda que si se escoge este tratamiento, la terapeuta proceda con un protocolo de trauma reciente para hacer blanco específicamente sobre los recuerdos traumáticos del reciente tornado. El segundo especialista, Andrew Leeds, comenta sobre la ausencia de ensayos controlados aleatorizados (ECA) u otros informes científicos que exploren la seguridad del tratamiento EMDR con mujeres embarazadas. Señala que las mujeres embarazadas con síntomas de estrés postraumático tendrían que entender que hay grandes posibilidades de que el EMDR mejore su calidad de vida como madre y que, probablemente, los riesgos de sufrir efectos adversos en la estabilidad del embarazo son muy bajos, aunque siguen siendo desconocidos. La tercera especialista, Claire Stramrood, explica que los pocos estudios de casos que han evaluado el EMDR durante el embarazo han encontrado efectos positivos, pero estaban relacionados con mujeres con trastorno de estrés postraumático (TEPT) posterior al nacimiento del bebé. Afirma que una vez que se haya consultado al obstetra, se haya informado a las mujeres de los posibles riesgos y beneficios y hayan firmado un consentimiento informado, deberían de poder decidir si comenzar la terapia EMDR durante el embarazo.
\end{abstract}

Palabras clave: EMDR; estrés postraumático; trastorno de estrés agudo; tornado; embarazo

\section{Pregunta del terapeuta}

Tengo una cliente "Judy", que me derivó su terapeuta principal para terapia de desensibilización y reprocesamiento por movimientos oculares (EMDR). Está embarazada de 7 meses. Hace unos meses estuvo en un tornado en el que su casa quedó completamente arrasada y el armario en el que ella y su familia estaban escondidos fue arrastrado por el tornado y "aterrizó” en la calle. Todos ellos resultaron heridos.

Judy se encuentra obsesionada, llena de pánico, asustada ... por mencionar algo ... por el tiempo. Ha comprado cascos para toda la familia. Cuando

This article originally appeared as Forgash, C., Leeds, A., Stramrood, C., \& Robbins, A. (2013). Case consultation: Traumatized pregnant woman. Journal of EMDR Practice and Research, 7(1), 45-49. Translated by Miriam Ramos Morrison. 
el tiempo se pone ligeramente lluvioso y tormentoso, llega a tener ataques de pánico y hace que todo el mundo se esconda, comprueba el tiempo varias veces al día y se vuelve hiperenfocada cuando se acerca el "mal tiempo" de forma inminente.

Judy oyó hablar de EMDR y está desesperada por hacerlo para aliviar estos problemas. Le expliqué la responsabilidad de hacerlo con una mujer embarazada y ella dijo: "No me preocupa, lo que le estoy haciendo a mi bebé (estrés extremo) es mucho peor que hacer EMDR”. Tengo un consentimiento informado. ¿Qué pensáis sobre esto? ¿Harías EMDR con ella?

\section{Respuesta de la especialista $n^{\circ} 1$, Carol Forgash}

Me gustaría empezar por aportar algunas observaciones generales sobre el embarazo y la psicoterapia. El embarazo se define como un estado de salud normal para la mayoría de las mujeres: llevar un embrión desde la concepción hasta el parto. Aunque, hasta el último cuarto del siglo XX, los médicos a menudo lo patologizaban, el embarazo no es una enfermedad.

Como terapeuta que ha tratado a muchas mujeres embarazadas, me siento cómoda cuando me planteo usar EMDR con clientes embarazadas. Sin embargo, en la comunidad de EMDR, he observado que el tratamiento de las mujeres embarazadas sigue siendo motivo de preocupación para muchos terapeutas. Baso esta declaración en el gran número y frecuencia de las preguntas que se plantean en el LISTSERV EMDR Institute sobre el uso de EMDR con pacientes embarazadas.

Si los terapeutas están dispuestos a ver un embarazo como una situación normal, es más fácil tener claridad en el juicio clínico. Esto nos lleva al tema de la contratransferencia. Si la terapeuta ha tenido un embarazo difícil, es posible que tenga que buscar supervisión porque su propio estado de ánimo puede influir en su decisión de usar el método EMDR con clientes embarazadas. Los terapeutas masculinos que tengan un cónyuge o pariente que pueda haber tenido problemas durante el embarazo también pueden beneficiarse de supervisar los temas que surgen para ellos. Proporcionar información sobre el método EMDR al obstetra o la partera y obtener su consentimiento también puede disipar las preocupaciones del terapeuta. También recomendaría que el terapeuta tuviera una conversación con el médico y le pidiera información sobre cualquier cuestión pertinente que pueda afectar al tratamiento. Además, el terapeuta puede dejar saber al médico que entiende las implicaciones del trastorno de estrés postraumático (TEPT) en la salud de la paciente y explicarle la necesidad de tratar del trauma.

En el caso de Judy, donde no tenemos mucha información previa al embarazo ni sobre su salud actual, diría lo siguiente: En circunstancias normales, si la cliente se encuentra en buen estado de salud, el embarazo es estable y el terapeuta ha recibido la aprobación del obstetra para tratarla con EMDR, yo confiaría y respetaría su decisión de someterse a tratamiento con EMDR. Un alto nivel de hormonas de estrés es perjudicial tanto para la mujer embarazada como para su bebé (Poggi-Davis y Sandman, 2006). Parece que Judy ha estado seriamente estresada desde el tornado y tiene síntomas intrusivos de TEPT. Está en lo cierto sobre la necesidad de resolución del trauma (Harvey y Ramírez, 2010) porque el TEPT puede tener efectos negativos en la salud materna y fetal. Al consultar con el médico, hazle saber que entiendes las implicaciones de TEPT en la salud de la paciente y explícale la necesidad de tratar del trauma.

Recopilaría cuidadosamente la historia clínica, incluido cualquier suceso sobre temas de salud o trauma del pasado, lo que incluye la fertilidad de Judy y su historial de embarazos a lo largo su vida, sus actitudes hacia el embarazo y cualquier síntoma de TEPT, trastorno de pánico, ansiedad y depresión. También sería importante buscar alguna contraindicación al tratamiento. Estas podrían incluir un trastorno disociativo inestable o una psicosis. Otro problema sería una falta de apoyo importante de la pareja / cónyuge / familia. Su relación con su médico / partera es también un elemento importante a tener en cuenta. En cuanto a problemas médicos, ¿tiene alguna condición que la ponga en un alto nivel de riesgo?

Con excepción de estas condiciones, y dado que queda muy poco tiempo de embarazo, yo procedería a desarrollar un plan de tratamiento que incluyera educación acerca de EMDR, enseñarle estrategias de autorrelajación y reducción de estrés (en caso necesario) y luego establecería un blanco relacionado con las experiencias vividas durante el tornado y las procesaría.

Si Judy quisiera continuar con el tratamiento después del parto, porque tuviera una historia de trauma previo importante, explicaría que se pueden tratar más adelante otros eventos traumáticos. El tratamiento actual se centraría en el tornado y los efectos que ha tenido sobre ella.

Además, debido a que el tornado ha ocurrido tan solo hace unos meses, trabajaría con un protocolo de incidente reciente de EMDR en lugar del protocolo estándar de EMDR. La continua experiencia de angustia de esta paciente relacionada con sus experiencias 
en el tornado indica claramente que los efectos del evento original han continuado hasta el día de hoy. Utilizaría el protocolo de episodio traumático reciente (PET-R; Shapiro y Laub, 2008). Se entiende que este protocolo integra partes no procesadas de un episodio traumático completo: el incidente, las experiencias que han ocurrido después de los hechos y el posible cambio de significado del incidente original hasta el presente. El PET-R trabaja con las imágenes perturbadoras, el evento, múltiples blancos dentro del evento y la temática de todo el episodio. Tiene el potencial de limitar las asociaciones a sólo este episodio al utilizar la desensibilización por movimientos oculares (EMD; Shapiro, 1989), en la cual se vuelve de manera repetida al blanco. El objetivo sería mantener las puertas cerradas a cualquier trauma anterior.

El PET-R también hace hincapié en las estrategias de preparación y de contención, como los ejercicios de autorrelajación y reducción de estrés mencionados anteriormente. En el enfoque PET-R, el cliente cuenta la historia cronológica del trauma mientras se usa estimulación bilateral. El siguiente paso es analizar el evento e informar de los puntos de perturbación (PDP). Se desarrolla un blanco (imagen, cognición negativa $[\mathrm{CN}]$, cognición positiva $[\mathrm{CP}]$, validez de la cognición [VOC], unidades subjetivas de perturbación [SUD], sensación corporal) en torno a cada PDP. Para Judy, posibles puntos de perturbación podrían ser la escena de la casa siendo arrasada, las lesiones de su familia o un día lluvioso. Se procesaría cada uno de ellos. Por último, se procesaría la temática de todo el episodio. Para esta cliente embarazada en particular, este enfoque sería muy apropiado.

\section{Respuesta del especialista $n^{\circ} 2$, Andrew M. Leeds}

La cuestión de la seguridad del EMDR en mujeres embarazadas no se ha abordado de manera explícita en la literatura científica. Las búsquedas en bases de datos en internet (PubMed, PsycINFO y la Biblioteca Francine Shapiro) no han mostrado informes publicados que indiquen que el tratamiento EMDR pueda suponer peligro alguno. Por el contrario, Stramrood et al. (2012) han indicado resultados clínicos positivos en una serie de casos con tres mujeres embarazadas que habían desarrollado síntomas de estrés postraumático después de experiencias traumáticas en su primer embarazo. En los tres casos, se les proporcionó tratamiento EMDR durante su segundo embarazo, en algunos casos derivadas a tratamiento por su obstetra.

Como formador de EMDR y durante los últimos 20 años, he recibido un pequeño número de informes personales aislados en los que algunas mujeres se han quejado de inestabilidad en su embarazo después de las sesiones de EMDR. Estas mujeres creían que el tratamiento con EMDR había sido un factor en su necesidad posterior de reposo y atención médica (Leeds, 2009, p. 88). Dichos informes no pueden considerarse pruebas científicas de que existan riesgos, pero tampoco deben desestimarse. Es posible que las mujeres que enviaron estos informes pudieran haber sufrido embarazos inestables incluso si no hubieran participado en las sesiones de tratamiento EMDR. En el transcurso de muchos grupos de consulta y talleres, he observado que el miedo a tratar con EMDR a mujeres embarazadas está bastante generalizado. Esto puede deberse a los posibles "factores de riesgo" que se han identificado en los manuales de los programas de formación estándar de EMDR desde 1991 en adelante. (Parecen que existen temores infundados similares respecto a ofrecer EMDR a aquellos que se enfrentan a procedimientos legales o a individuos con ataques epilépticos auténticos). Por lo tanto, un terapeuta que crea erróneamente que EMDR es demasiado "peligroso" para una mujer embarazada puede que no anime a su paciente a consultar con su obstetra.

Se sabe que el tratamiento EMDR es altamente eficaz y eficiente para los síntomas de estrés postraumático (Bisson y Andrew, 2007). Ante la falta de estudios científicos sobre la seguridad del tratamiento EMDR para mujeres embarazadas, se han de sopesar los probables beneficios en la calidad de vida de la madre (y la reducción de estrés para el feto en desarrollo) frente a un desconocido nivel de seguridad. Hasta que haya ECA con muestras suficientemente grandes de mujeres embarazadas, un debate adecuado indicaría que el riesgo para la estabilidad del embarazo probablemente sea bajo, pero puede que no sea cero, mientras que los beneficios potenciales y probables para las mujeres con síntomas de estrés postraumático (y sus fetos) son altos. Hasta que haya ECA que proporcionen mayor orientación sobre este tema, un enfoque conservador sugeriría que el reprocesamiento EMDR sólo debe emplearse con mujeres embarazadas después de haber consultado con el médico de la mujer o si éste nos la ha derivado. En la actualidad, parece que se aplican los mismos riesgos generales a la hora de ofrecer tratamiento EMDR a mujeres embarazadas y al resto de adultos. Debería tener lugar, como siempre, una detección adecuada de factores de riesgo, tales como un trastorno disociativo grave. En el caso de Judy, la información que se ha proporcionado en el resumen es insuficiente para determinar claramente su nivel de preparación para EMDR. En concreto, no se proporciona información 
acerca de su historia temprana, si existe un historial de trastornos de ansiedad o si el terapeuta ha detectado síntomas disociativos. En una verdadera supervisión de caso de EMDR, se solicitaría dicha información. Una vez que se hayan aclarado estas cuestiones y se haya obtenido un consentimiento informado, se debe ofrecer EMDR a las mujeres que estén o puedan estar embarazadas cuando estén sufriendo de síntomas para los que EMDR es conocido por su eficacia.

Judy está deseosa de hacer EMDR. No existen riesgos identificados y definidos para el tratamiento EMDR de Judy o de mujeres embarazadas en general. Dada la ausencia de factores de riesgo específicos que no se han detallado en el resumen del caso, sería una pena prolongar su sufrimiento por el mero hecho de que está embarazada. Por otra parte, en el momento del parto, sus síntomas no tratados de TEPT podrían afectar negativamente a su capacidad para establecer el vínculo materno-infantil (Liotti, 1992; Madrid, Skolek, y Shapiro, 2006), lo que complicaría su tratamiento posterior y podría afectar a su hijo recién nacido. En mi propia práctica clínica, en el supuesto de que cumpliera con los criterios generales de preparación, la respuesta es "sí". Yo ofrecería tratamiento EMDR a Judy. En ausencia de una derivación de su obstetra (o antecedentes de embarazo inestable), primero le sugeriría que hablase con su médico sobre su deseo de recibir tratamiento EMDR, pero tanto si tuviese esa conversación como si no, los beneficios del tratamiento EMDR son muy superiores a la riesgos generales de tratamiento.

\section{Respuesta de la especialist $n^{\circ} 3$, Claire A. I. Stramrood}

En muchos casos de problemas psicológicos durante el embarazo, incluidos estrés, depresión y ansiedad, los médicos y terapeutas se enfrentan a escoger entre dos males: usar tratamientos potencialmente dañinos para el feto o prolongar el estado mentalmente enfermo con posibles efectos adversos también para el feto (y la mujer embarazada).

En mi respuesta a esta consulta sobre el caso, voy a presentar varios argumentos a favor del uso de EMDR durante el embarazo. Sin embargo, en este caso particular, hablaría con Judy de los riesgos y beneficios que conllevaría y luego dejaría la decisión en sus manos.

En las pautas de práctica internacionales y nacionales, se recomiendan la terapia cognitivo-conductual centrada en el trauma y el EMDR como tratamientos de elección para víctimas de trauma que sufren de trastorno de estrés postraumático o trastorno de estrés agudo (American Psychiatric Association, 2004; National
Institute for Health and Clinical Excellence [NICE], 2005). La información aportada para este caso es escasa, pero incluye características de ambas condiciones.

Un primer paso necesario sería un diagnóstico adecuado de los síntomas de Judy. Una vez que se haya establecido que sus síntomas son tales que podría beneficiarse de EMDR, la pregunta es si el tratamiento debería aplicarse durante su embarazo. En el libro de EMDR de 2001, Shapiro recomienda precaución porque una posible hiperactivación y aumento de la tensión después del tratamiento podrían afectar negativamente a la madre y al feto (p. 96).

Desafortunadamente, no se han investigado bien los riesgos y beneficios de aplicar EMDR durante el embarazo. Se han publicado dos estudios piloto relacionados con mujeres con TEPT después del parto. Un estudio utilizó EMDR con tres mujeres embarazadas (Stramrood et al., 2012) y en otro estudio se estudiaron cuatro mujeres de las cuales una estaba embarazada (Sandstrom, Wilberg, Wikman, Willman y Hogberg, 2008). Los estudios estaban relacionados con mujeres que habían sufrido TEPT después del parto. En otras palabras, el parto previo había sido el evento traumático.

El primer estudio se realizó en un grupo de investigación del que soy parte y tuvo lugar en un hospital universitario en Holanda. Las tres mujeres habían resultado traumatizadas durante el parto y cada una había desarrollado TEPT en relación al nacimiento de su hijo. Ahora estaban embarazadas de nuevo, mostraban síntomas de TEPT y expresaban sus temores sobre el futuro nacimiento. EMDR dio como resultado una reducción del estrés, menos síntomas de estrés postraumático y un aumento de su confianza sobre el parto de su segundo hijo. Por otra parte, a pesar de las complicaciones durante su segundo parto, las tres mujeres habían tenido una experiencia positiva del segundo nacimiento. Con las mujeres mencionadas previamente, habíamos tenido o bien una derivación directa o una consulta con el obstetra y, en mi opinión, este es un elemento fundamental.

En este caso específico, el trauma y el estrés de Judy no están relacionados con el embarazo o el parto, sino con un tornado que sufrió su familia. Teniendo en cuenta el hecho de que Judy ya está embarazada de 7 meses, puedo entender argumentos para posponer el tratamiento EMDR hasta después de que el bebé haya nacido. Se sabe que el estrés afecta de forma negativa al desarrollo neurológico del feto (O’Donnel, O’Connor y Glover, 2009), pero con toda probabilidad, la mayoría de los efectos adversos del estrés de Judy en el feto ya han ocurrido a los 7 meses de gestación. Otro argumento sería que el propio EMDR puede provocar 
estrés, con posibles consecuencias negativas para el embarazo, como ha mencionado Shapiro (2001). Sin embargo, que el tratamiento haya tenido lugar o no durante el embarazo, se vuelve relevante en el periodo postparto debido a que el trauma / TEPT no resuelto puede afectar negativamente al vínculo entre la madre y el bebé (Parfitt y Ayers, 2009) y afectar al apego seguro del niño (Liotti, 1992).

Desde la perspectiva de Judy, puedo imaginar que experimentar diariamente tanto estrés relacionado con el tiempo es una carga para ella y cualquier solución con efectos potencialmente rápidos es más que bienvenida. Con esto en mente, definitivamente EMDR sería una opción, porque muchas personas experimentan efectos positivos tan sólo después de unas pocas sesiones.

En conclusión, el EMDR durante el embarazo hasta ahora se ha estudiado a pequeña escala, con efectos muy positivos para las mujeres que sufren de TEPT después de un parto anterior. En dichos casos, definitivamente recomendaría EMDR. Aunque no hay investigación sobre el tratamiento con EMDR durante el embarazo para aquellas mujeres cuyo trauma no está relacionado con un embarazo y parto anterior, la causa del TEPT no es importante y esta es razón insuficiente para disuadir o rechazar el EMDR durante el embarazo. Si habido una derivación del obstetra o se le ha consultado, se ha informado a las mujeres de los riesgos y beneficios y han dado su consentimiento informado, deberían ser capaces de tomar la decisión ellas mismas.

\section{Referencias}

American Psychiatric Association. (2004). Practice guidelines for the treatment of patients with acute stress disorder and posttraumatic stress disorder. Arlington, VA: Author.

Bisson, J., \& Andrew, M. (2007). Psychological treatment of post-traumatic stress disorder (PTSD). Cochrane Database of Systematic Reviews, (3), CD003388.

Harvey, L., \& Ramirez, A. (2010). Hurricane trauma: The effects of prenatal stress on child development. Retrieved from http: / / uwf.edu/argojournal/admin/body/Research_ Paper-Harvey_\&_Ramirez_Submission_for_Argo_ Journal.pdf

Leeds, A. M. (2009). A guide to the standard EMDR protocols for clinicians, supervisors, and consultants. New York, NY: Springer Publishing.

Liotti, G. (1992). Disorganized/disoriented attachment in the etiology of the dissociative disorders. Dissociation, 5(4), 196-204.

Madrid, A., Skolek, S., \& Shapiro, F. (2006). Repairing failures in bonding through EMDR. Clinical Case Studies, 5(4), 271-286.
National Institute for Health and Clinical Excellence. (2005). Post-traumatic stress disorder: The management of PTSD in adults and children in primary and secondary care. London, United Kingdom: Author.

O’Donnell, K., O’Connor, T. G., \& Glover, V. (2009). Prenatal stress and neurodevelopment of the child: focus on the HPA axis and role of the placenta. Developmental Neuroscience, 31(4), 285-292.

Parfitt, Y. M., \& Ayers, S. (2009). The effect of post-natal symptoms of post-traumatic stress and depression on the couple's relationship and parent-baby bond. Journal of Reproductive and Infant Psychology, 27(2), 127-142.

Poggi-Davis, E., \& Sandman, C. A. (2006). Prenatal exposure to stress and stress hormones influences child development. Infants and Young Children, 19(3), 246-259.

Sandstrom, M., Wiberg, B., Wikman, M., Willman, A. K., \& Hogberg, U. (2008). A pilot study of eye movement desensitisation and reprocessing treatment (EMDR) for posttraumatic stress after childbirth. Midwifery, 24(1), 62-73.

Shapiro, F. (1989). Eye movement desensitization: A new treatment for post-traumatic stress disorder. Journal of Behavior Therapy and Experimental Psychiatry, 20, 211-217.

Shapiro, F. (2001). Eye movement desensitization and reprocessing. Basic principles, protocols, and procedures (2nd ed.). New York, NY: Guilford Press.

Shapiro, E., \& Laub, B. (2008). Early EMDR intervention (EEI): A summary, a theoretical model, and the recent traumatic episode protocol (R-TEP). Journal of EMDR Practice and Research, 2(2), 79-96.

Stramrood, C. A. I., van der Velde, J., Doornbos, B., Paarlberg, K. M., Weijmar Schultz, W. C. M., \& van Pampus, M. G. (2012). The patient observer: Eye-movement desensitization and reprocessing for the treatment of posttraumatic stress following childbirth. Birth, 39 (1), 70-76.

\section{Conoce a los especialistas}

Carol Forgash es trabajadora social clínica licenciada en consulta privada en Smithtown, New York y está especializado en trauma complejo. Es consultora aprobada por EMDR International Association (EMDRIA) y también ofrece talleres y cursos aprobados por EMDRIA. Ha coeditado el libro Healing the Heart of Trauma and Dissociation with EMDR and Ego State Therapy y ha escrito diversos artículos y capítulos en libros sobre EMDR. Ex ex presidente del programa de ayuda humanitaria de EMDR (HAP).

El Dr. Andrew M. Leeds, PhD, es psicólogo licenciado en California y director de formación en el Sonoma Psychotherapy Training Institute de Santa Rosa, California. Es consultor aprobado por EMDRIA y EMDR Europa y ofrece formación básica en EMDR. Es el autor de Guía de protocolos estándar de EMDR para terapeutas, supervisores y consultores (2013) y ha escrito 
diversos capítulos en libros y artículos sobre EMDR, incluido un estudio de investigación sobre desarrollo e instalación de recursos (DIR).

La Dra. Claire A. I. Stramrood, MD, es médico residente en ginecología / obstetricia en Utrecht, Holanda. Ha terminado recientemente una tesis doctoral sobre mujeres con TEPT después del parto. Fue la autora principal de un estudio piloto sobre el tratamiento con EMDR para el TEPT después del parto que se publicó en 2012. También se ha implicado en el establecimiento de un modelo de cuidados integrales para mujeres con miedo a dar a luz.

La correspondencia sobre este artículo debería dirigirse a Carol Forgash, LCSW, BCD, 353 North Country Road, Smithtown, NY 11787. E-mail: cforgash@optonline.net 